\title{
Evidence for Second-Phonon Nuclear Wobbling
}

D. R. Jensen, ${ }^{1}$ G. B. Hagemann, ${ }^{1}$ I. Hamamoto, ${ }^{1,2}$ S. W. Ødegård, ${ }^{1,3}$ B. Herskind,,${ }^{1}$ G. Sletten, ${ }^{1}$ J. N. Wilson, ${ }^{1}$ K. Spohr, ${ }^{4}$ H. Hübel, ${ }^{5}$ P. Bringel,${ }^{5}$ A. Neußer, ${ }^{5}$ G. Schönwaßer, ${ }^{5}$ A. K. Singh, ${ }^{5}$ W. C. Ma, ${ }^{6}$ H. Amro, ${ }^{6}$ A. Bracco, ${ }^{7}$ S. Leoni, ${ }^{7}$ G. Benzoni, ${ }^{7}$ A. Maj, ${ }^{8}$ C. M. Petrache, ${ }^{9,10}$ G. Lo Bianco, ${ }^{10}$ P. Bednarczyk,${ }^{8,11}$ and D. Curien ${ }^{11}$

${ }^{1}$ The Niels Bohr Institute, Blegdamsvej 17, DK-2100 Copenhagen Ø, Denmark

${ }^{2}$ Department of Mathematical Physics, LTH, University of Lund, Lund, Sweden ${ }^{3}$ Department of Physics, University of Oslo, PB 1048 Blindern, N-0316 Oslo, Norway

${ }^{4}$ Department of Electronic Engineering and Physics, University of Paisley, Scotland

${ }^{5}$ ISKP, University of Bonn, Nussallee 14-16, D-53115 Bonn, Germany

${ }^{6}$ Mississippi State University, Mississippi State, MS 39762

${ }^{7}$ Dipartemento di Fisica and INFN, Sezione di Milano, Milano, Italy

${ }^{8}$ Niewodniczanski Institute of Nuclear Physics, Krakow, Poland

${ }^{9}$ Dipartemento di Fisica and INFN, Sezione di Padova, Padova, Italy

${ }^{10}$ Dipartimento di Matematica e Fisica, Universita di Camerino, Camerino, Italy

${ }^{11}$ IReS, 23 rue du Loess, BP28 F-67037, Strasbourg, France

(Received 17 May 2002; published 16 September 2002)

\begin{abstract}
The nucleus ${ }^{163} \mathrm{Lu}$ has been populated through the reaction ${ }^{139} \mathrm{La}\left({ }^{29} \mathrm{Si}, 5 n\right)$ with a beam energy of $157 \mathrm{MeV}$. Three triaxial, strongly deformed (TSD) bands have been observed with very similar rotational properties. The first excited TSD band has earlier been assigned as a one-phonon wobbling excitation built on the lowest-lying (yrast) TSD band. The large $B(E 2)_{\text {out }} / B(E 2)_{\text {in }}$ value obtainable for one of four observed transitions between the second and first excited TSD bands is in good agreement with particle-rotor calculations for a two-phonon wobbling excitation.
\end{abstract}

DOI: $10.1103 /$ PhysRevLett.89.142503

PACS numbers: 27.70.+q, 21.10.Re, 23.20.En

The ground states of some nuclei are spherical, while others are deformed. The most important deformations around the ground states of nuclei are known to be of axial symmetric quadrupole type, with no clear evidence for the presence of axial asymmetry, i.e., triaxiality. On the other hand, at high spins one expects a considerable deviation from the axial symmetric shape which is naturally caused by the Coriolis and centrifugal forces.

The rotational motion of a triaxial deformed nucleus with three different moments of inertia, $J_{x}>$ $J_{y} \neq J_{z}$ induces in the high-spin limit, $I \gg 1 \hbar$, a sequence of wobbling bands described by the energy, $E_{R}\left(I, n_{w}\right)=I(I+1) / 2 J_{x}+\hbar \omega_{w}\left(n_{w}+1 / 2\right)$, where $n_{w}$ is the wobbling phonon number, and $\hbar \omega_{w}=$ $\hbar \omega_{\text {rot }} \sqrt{\left(J_{x}-J_{y}\right)\left(J_{x}-J_{z}\right) /\left(J_{y} J_{z}\right)}$ with $\hbar \omega_{\text {rot }}=I / J_{x}$ [1]. A family of wobbling bands is expected to have very similar intrinsic structure. Each band, characterized by the quantum number $n_{w}=1,2, \ldots$, can be seen as a wobbling phonon excitation built on the yrast $n_{w}=0$ band. A characteristic signature of the wobbling motion is the occurrence of $\Delta I= \pm 1 \hbar$ interband transitions, $\left(n_{w}=i\right) \rightarrow\left(n_{w}=i-1\right)$ with $i=1,2, \ldots$, possessing large values of $B(E 2)_{\text {out }}$, in competition with $B(E 2)_{\text {in }}$ of the inband $\Delta I=2 \hbar$ transitions. The quantal phonon rule for transition probability implies that $B\left(E 2, n_{w}=2 \rightarrow n_{w}=1\right)=2 B\left(E 2, n_{w}=1 \rightarrow n_{w}=0\right)$ and that transitions with $\Delta n_{w}=2$ are forbidden. The observation of wobbling phonon excitations is uniquely related to the triaxiality of the system. In the $N \sim 94$ and $Z \sim 71$ region "ultimate cranker" (UC) calculations [2], based on a modified harmonic oscillator potential, predict stable triaxial minima in the total potential energy surface with $(\epsilon, \gamma) \approx\left(0.38, \pm 20^{\circ}\right)$ for all pairs of parity and signature, $(\pi, \alpha)$. In ${ }^{163} \mathrm{Lu}$ two excited triaxial strongly deformed (TSD) bands have previously been observed to decay to the yrast $\pi i_{13 / 2}$ TSD band (TSD1) [3]. The first excited TSD band (TSD2), that decays to TSD1 via $9 \Delta I=1 \hbar$ transitions, has recently been assigned as a one-phonon wobbling excitation built on the yrast TSD band [4]. The wobbling degree of freedom applies to the rotational angular momentum $\vec{R}$ of the triaxial core while the angular momentum $\vec{j}$ of the $i_{13 / 2}$ proton remains fully aligned. The firm evidence for the interpretation of TSD2 as a one-phonon wobbling excitation was provided by the electromagnetic transition matrix elements of the connecting transitions between TSD2 and TSD1 in comparison with calculations. In particular, the large $B(E 2)_{\text {out }} / B(E 2)_{\text {in }}$ values are not consistent with a configuration based on the signature partner or a possible pair of two-quasineutrons coupled to the $i_{13 / 2}$ quasiproton. In our previous paper [3] another excited band, TSD3, was found to decay to TSD1 through 5 $\Delta I=2 \hbar$ transitions. TSD3 was also observed to be in coincidence with TSD2 but no transitions could be firmly established between the two bands. Based on this observation TSD3 was considered a possible candidate for a two-phonon wobbling excitation also built on TSD1. The observation of $\Delta I=1 \hbar$ transitions from TSD3 to TSD2 with large $B(E 2)$ values, is crucial for the assignment in the present work of TSD3 as a two-phonon wobbling 
excitation based on a comparison with particle-rotor calculations.

To find and investigate the properties of the transitions from TSD3 to TSD2 the nucleus ${ }^{163} \mathrm{Lu}$ was populated through the fusion-evaporation reaction ${ }^{139} \mathrm{La}\left({ }^{29} \mathrm{Si}, 5 n\right)$ with a beam energy of $157 \mathrm{MeV}$ provided by the Vivitron accelerator at IReS, Strasbourg. Approximately $6 \times 10^{9} \gamma$ ray coincidence events were collected with the Euroball IV detector array [5] consisting of 15 cluster, 25 clover, and 27 tapered Compton-suppressed Ge detectors together with a BGO inner ball used as a multiplicity filter. The data were sorted into a $\gamma^{3}$ coincidence matrix for coincidence analysis [6]. For the analysis of directional correlation of $\gamma$ rays from the oriented states (DCO) and angular distributions, the data was sorted into three types of 2D matrices with the different detector angle combinations on the two axes, (all angles vs $90^{\circ}$ positions), (all angles vs $25^{\circ}$ positions) and $\left(90^{\circ}\right.$ vs $25^{\circ}$ positions) in a similar way as in Refs. [3,4].

The three previously known TSD bands with relative populations of $\sim 10,3$, and $1.2 \%$ of yrast are shown in Fig. 1 together with the normal deformed (ND) states to which they decay. TSD3 could be extended by one transition, now reaching spin $85 / 2 \hbar$. Four $\Delta I=1 \hbar$ transitions have been found between TSD3 and TSD2 in the spin range $45 / 2$ to $33 / 2 \hbar$ and eight $\Delta I=2 \hbar$ transitions have been firmly established between TSD3 and TSD1 covering the spin range from $61 / 2$ to $33 / 2 \hbar$. Spectra documenting the decayout transitions of TSD3 are displayed in Fig. 2. In the upper spectrum the strongest transition at $476 \mathrm{keV}$ is clearly seen whereas the $445 \mathrm{keV}$ transition is contaminated with a normal deformed $\gamma$ ray of the same energy. The two weaker decayout transitions at 411 and $377 \mathrm{keV}$ are also indicated.

In order to determine the multipolarity of the decayout transitions from TSD3 a spin alignment of $\sigma / I=0.25 \pm$ 0.02 was deduced using the same method as described in Refs. [3,4]. The $\sigma / I$ ratio remains constant in the spin range from $61 / 2$ to $29 / 2 \hbar$. Stretched $E 2$ character (disregarding $M 2$ ) has been assigned to the transitions from TSD3 to TSD1 based on the angular distribution ratios, $W\left(25^{\circ}\right) / W\left(90^{\circ}\right)$, and DCO-ratios shown in Table I. For the transitions from TSD3 to TSD2, $W\left(25^{\circ}\right) / W\left(90^{\circ}\right)$ and DCO-ratios could only be measured for the $476 \mathrm{keV} \gamma$ ray.

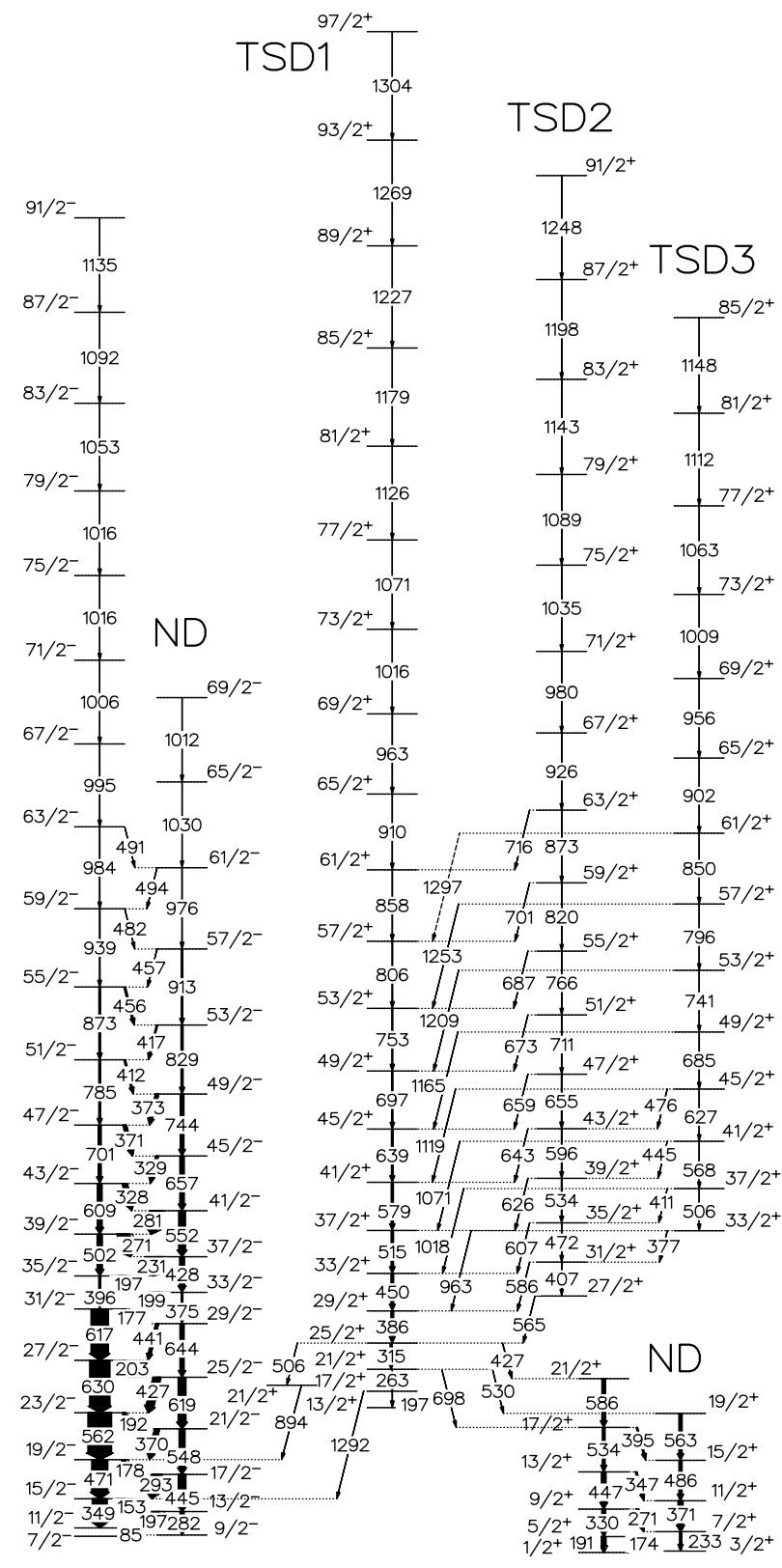

FIG. 1. Partial level scheme of ${ }^{163} \mathrm{Lu}$.

TABLE I. Experimental values of branching ratio $\lambda$, angular distribution ratio $W\left(25^{\circ}\right) / W\left(90^{\circ}\right)$, DCO-ratio, and $B(E 2)_{\text {out }} / B(E 2)_{\text {in }}$ for transitions from TSD3 to TSD1.

\begin{tabular}{ccccc}
\hline \hline$E_{\gamma}(\mathrm{keV})$ & $\lambda$ & $\frac{W\left(25^{\circ}\right)}{W\left(90^{\circ}\right)}$ & DCO-ratio $^{\mathrm{a}}$ & $\frac{B(E 2)_{\text {out }}}{B(E 2)_{\text {in }}}$ \\
\hline 1018.1 & $0.71 \pm 0.13$ & $1.41 \pm 0.15$ & & $0.021 \pm 0.004$ \\
1070.8 & $0.52 \pm 0.07$ & & & $0.022 \pm 0.003$ \\
1119.2 & $0.33 \pm 0.05$ & $1.49 \pm 0.08$ & & $0.018 \pm 0.003$ \\
1165.3 & $0.24 \pm 0.07$ & $1.44 \pm 0.10$ & $1.01 \pm 0.15$ & $0.017 \pm 0.004$ \\
1209.5 & $0.26 \pm 0.06$ & $1.46 \pm 0.10$ & $1.04 \pm 0.15$ & $0.022 \pm 0.005$ \\
1253.8 & $0.19 \pm 0.05$ & & & $0.020 \pm 0.005$ \\
\hline \hline
\end{tabular}

${ }^{\mathrm{a}}$ Gated on stretched E2 transitions. 


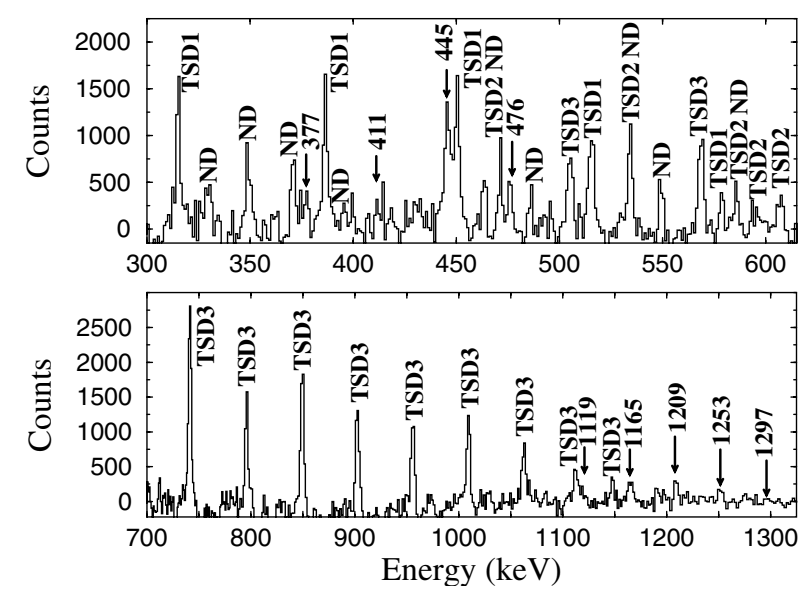

FIG. 2. Upper panel: Low energy part of a spectrum obtained by a sum of double gates on energies $315,386,450$, and $515 \mathrm{keV}$ in TSD1 vs 685 and $741 \mathrm{keV}$ in TSD3 showing the decay of TSD3. The $\Delta I=1 \hbar$ transitions from TSD3 to TSD2 are marked by arrows. Lower panel: Same as above but with the gates in TSD3 at 796, 850, 902 and $956 \mathrm{keV}$ showing transitions in TSD3 together with the $\Delta I=2 \hbar$ transitions to TSD1 marked by arrows.

The results are given in Table II and are in good agreement with expectations for a mixed $E 2 / M 1 \Delta I=1 \hbar$ transition. From these results spin and parity of the states in TSD3 have been firmly determined. The two solutions for the mixing ratio, $\delta$, each obtained as an average of the values extracted from the angular distribution and DCOratios are also listed in Table II. A linear polarization analysis of the $476 \mathrm{keV} \gamma$ ray was impossible due to low statistics. A selection of one of the two experimental $\delta$ values could therefore not be made on experimental grounds.

Using the particle-rotor model in which one $i_{13 / 2}$ quasiproton is coupled to the core of triaxial shape spectroscopic properties of the states in the yrast region have been studied [7]. When the parameters are chosen so as to be appropriate for the high-spin TSD bands of ${ }^{163} \mathrm{Lu}$, one finds that four bands (the lowest and second lowest favored bands, $f 1$ and $f 2$, and the lowest and third lowest unfavored bands, $u 1$ and $u 3$ ) out of the lowest-lying six bands (three $\alpha_{f}$ and three $\alpha_{u}$ ) are identified to form a family of wobbling bands. As seen from Fig. 3, the nature of this family of bands can be found partly by analyzing

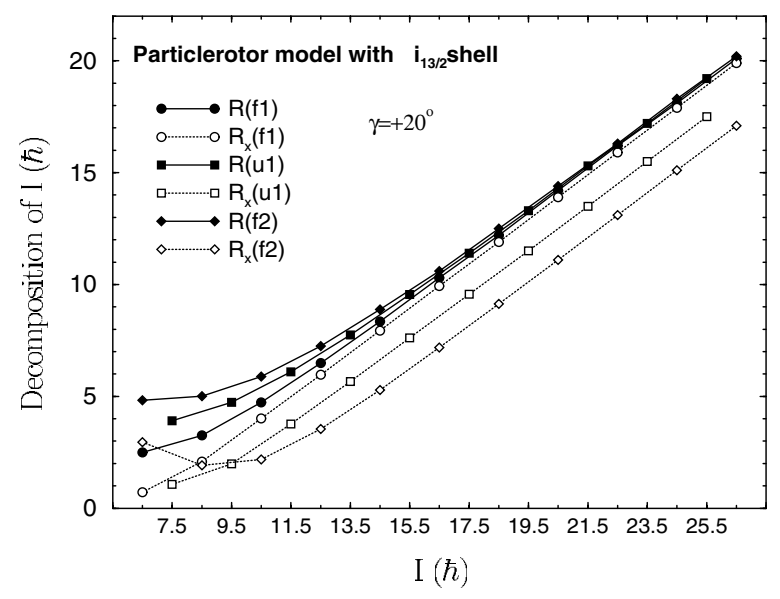

FIG. 3. Calculated values of $R$ and $R_{x}$ as a function of the total angular momentum $I$.

the collective angular momentum $\vec{R}$ of the core $(\vec{R}+\vec{j}=$ $\vec{I})$ and its $x$ component $R_{x}$. Namely, the magnitudes of $\vec{R}$ for a given $I$ are almost the same for those bands, while the direction of $\vec{R}$ is tilted from the $x$ axis more and more, as one goes from the yrast band $f 1\left(n_{w}=0\right)$ to higherlying bands, $u 1\left(n_{w}=1\right)$ and $f 2\left(n_{w}=2\right)$. The calculated particle alignments for a given $I$ are very similar for the bands $f 1, u 1$, and $f 2$ [7].

In Fig. 4 the experimental dynamic moment of inertia and alignment of TSD2 and TSD3 relative to TSD1 are shown in the upper and lower panel, respectively. The dynamic moment of inertia of TSD2 and TSD3 are almost identical over a wide spin range. Also the relative alignments behave in the same way as a function of frequency with a difference of only $\sim 0.1 \hbar$ between the two bands. Altogether this strongly suggests that TSD2 and TSD3 belong to the same family of bands with a very similar intrinsic structure. Based on the striking similarities between TSD3 and TSD2 the most likely value of $\delta$ for the transition from TSD3 to TSD2 is $\delta=-3.60_{-1.93}^{+0.97}$, very close to the previously found value for the transitions from TSD2 to TSD1 [3], see Table II. This solution corresponds to $\left(92.8_{-5.5}^{+4.0}\right) \%$ E2 and $\left(7.2_{-4.0}^{+5.5}\right) \% \quad M 1$ resulting in a large $B(E 2)_{\text {out }} / B(E 2)_{\text {in }}=0.51 \pm 0.13$, and $B(M 1)_{\text {out }} / B(E 2)_{\text {in }}=0.006_{-0.004}^{+0.006}\left(\mu_{N}^{2} / e^{2} b^{2}\right)$.

The experimental $B(E 2)_{\text {out }} / B(E 2)_{\text {in }}$ values for the $\Delta I=1 \hbar$ transitions from TSD2 to TSD1 and TSD3 to

TABLE II. Experimental values of branching ratio $\lambda$, angular distribution ratio $W\left(25^{\circ}\right) / W\left(90^{\circ}\right)$, DCO ratio, mixing ratio $\delta$, and $B(E 2)_{\text {out }} / B(E 2)_{\text {in }}$ for $\Delta I=1 \hbar$ transitions. Upper part: The $476 \mathrm{keV}$ transition from TSD3 to TSD2. Lower part: Average values for transitions from TSD2 to TSD1.

\begin{tabular}{ccccc}
\hline \hline$\lambda$ & $\frac{W\left(25^{\circ}\right)}{W\left(90^{\circ}\right)}$ & DCO-ratio $^{\mathrm{a}}$ & $\langle\delta\rangle$ & $\frac{B(E 2)_{\text {out }}}{B(E 2)_{\text {in }}}$ \\
\hline $0.14 \pm 0.04$ & $0.49 \pm 0.10$ & $0.38 \pm 0.11$ & $-3.60_{-1.93}^{+0.97}$ & $0.51 \pm 0.13$ \\
$\cdots$ & $\cdots$ & $\cdots$ & $-0.19_{-0.12}^{+0.08}$ & $0.019_{-0.017}^{+0.024}$ \\
\hline & $0.46 \pm 0.05$ & $0.33 \pm 0.03$ & $-3.10_{-0.44}^{+0.36}$ & $0.21 \pm 0.01$ \\
\hline \hline
\end{tabular}

\footnotetext{
${ }^{\mathrm{a}}$ Gated on stretched $E 2$ transitions.
} 


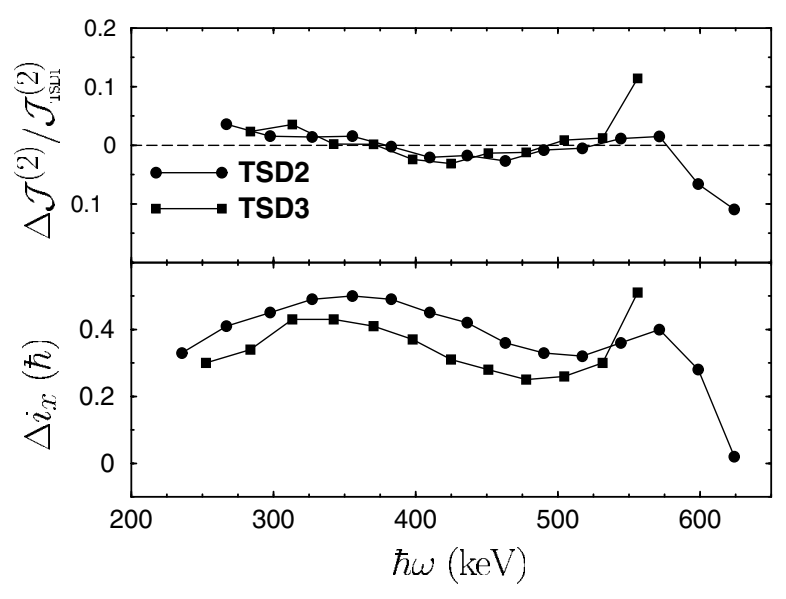

FIG. 4. Dynamic moment of inertia and alignment of TSD2 and TSD3 relative to TSD1 with $\Delta J^{(0}=J^{(2)}-J^{(2)}$ (TSD1) and $\Delta i_{x}=i_{x}-i_{x}$ (TSD1) using a rigid reference with $J_{\text {ref }}=$ $62 \hbar^{2} \mathrm{MeV}^{-1}$ as a function of frequency, $\hbar \omega$.

TSD2 as well as the $\Delta I=2 \hbar$ transitions from TSD3 to TSD1 are presented in Fig. 5 together with the theoretical results from the particle-rotor calculations. Although the large value quoted for the $B(E 2)_{\text {out }} / B(E 2)_{\text {in }}$ ratio for the $476 \mathrm{keV}$ transition from TSD3 to TSD2 has a large error its magnitude is consistent with the theoretical results for a two-phonon wobbling excitation. Also the experimental results obtained for the one-phonon excitation are in good agreement with the calculations [4], and the pure phonon behavior is almost realized. The $B(E 2)_{\text {out }} / B(E 2)_{\text {in }} \sim 0.02$ for the $\Delta I=2 \hbar$ transitions from TSD3 to TSD1, see Table I, arising from anharmonicities in the wobbling picture, are very close to the theoretical predictions as well.

It is known that for $j \ll R$ the anisotropy of the $g$ factor of the core is needed in order to obtain nonvanishing $M 1$ matrix elements in the $\Delta n_{w}=1$ transitions. Thus, the calculated $M 1$ matrix elements are anyway small. The calculated $B(M 1) / B(E 2)_{\text {in }}$ for the $45 / 2$ to $43 / 2 \hbar$ transition is $0.026\left(\mu_{N}^{2} / e^{2} b^{2}\right)$, which is more than a factor of 2 larger than the experimental upper limit of 0.012 $\left(\mu_{N}^{2} / e^{2} b^{2}\right)$. However, the inclusion of the presence (gradual increase) of neutron alignment in the core, which is observed but not included in the model of Ref. [7], will decrease the calculated $B(M 1)$ values.

Based on the almost identical rotational properties of TSD2 and TSD3 and the good agreement between the theoretical and experimental $B(E 2)_{\text {out }} / B(E 2)_{\text {in }}$ value of the $476 \mathrm{keV}$ transition from TSD3 to TSD2, TSD3 has been assigned as a two-phonon wobbling excitation.

The alternative solution for the mixing ratio, $\delta=$ $-0.19_{-0.12}^{+0.08}$, corresponds to $\left(3.5_{-2.3}^{+5.3}\right) \% \quad$ E2 and $\left(96.5_{-5.3}^{+2.3}\right) \% M 1$, giving $B(E 2)_{\text {out }} / B(E 2)_{\text {in }}=0.019_{-0.017}^{+0.024}$ and $B(M 1)_{\text {out }} / B(E 2)_{\text {in }}=0.083 \pm 0.024\left(\mu_{N}^{2} / e^{2} b^{2}\right)$. We find no band, of which the electromagnetic properties are consistent with this solution of $\delta$, among the lowestlying $\alpha_{f}$ bands obtained in the calculation of Ref. [7]. Moreover, adopting this second solution would imply that

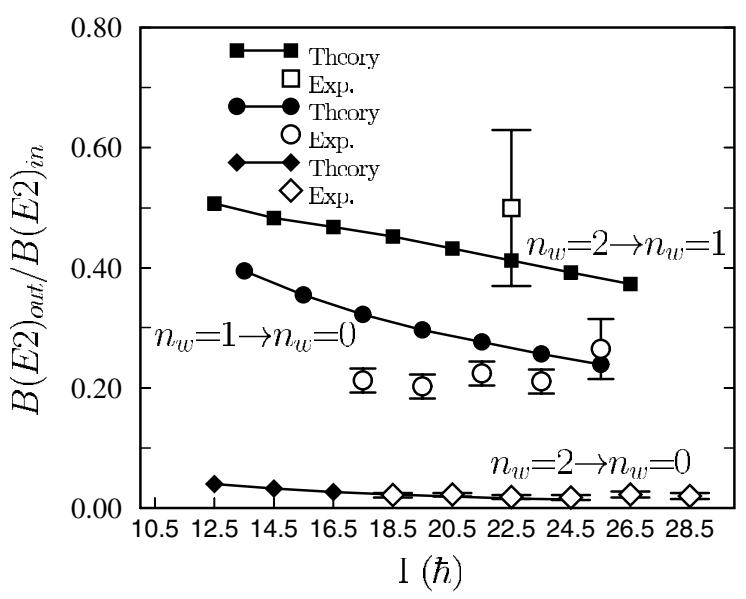

FIG. 5. Experimental and theoretical values of $B(E 2)_{\text {out }} / B(E 2)_{\text {in. }}$ TSD1, TSD2, and TSD3 are identified with $n_{w}=0,1$, and 2 , respectively.

TSD3 has an intrinsic structure quite different from that of TSD1 and TSD2, which in turn would lead to values of alignment and moment of inertia different from those observed in TSD1 and TSD2.

In conclusion, the second excited TSD band (TSD3) in ${ }^{163} \mathrm{Lu}$ has been connected to the first excited one-phonon wobbling excitation, TSD2, by $4 \Delta I=1 \hbar$ transitions. Spin and parity of TSD3 have been firmly determined. The $B(E 2)_{\text {out }} / B(E 2)_{\text {in }}$ value has been measured for one of the four transitions and found to be consistent with theoretical particle-rotor calculations for a two-phonon wobbling excitation. TSD3 has therefore been assigned as a two-phonon wobbling excitation built on the yrast TSD band fulfilling the phonon rule. The nucleus has revealed its exploitation of the quantal wobbling degree of freedom. This proves the triaxial deformation of the nucleus and adds a new dimension to the description of a rotating nucleus.

This research is supported by EU, Contract No. HPRICT-1999-00078, the EU TMR network project, Contract No. ERBFMRXCT970123, the Danish Science Foundation, the Research Council of Norway, the German BMBF (Contract No. 06 BN 907), the Polish State Committee for Scientific Research (KBN Grant No. 2 P03B 118 22), Crafoordska stiftelsen and the A.v. Humboldt Foundation.

[1] A. Bohr and B. R. Mottelson, Nuclear Structure (Benjamin, New York, 1975), Vol. II.

[2] T. Bengtsson, Nucl. Phys. A496, 56 (1989); A512, 124 (1990).

[3] D. R. Jensen et al., Nucl. Phys. A703, 3 (2002).

[4] S.W. Ødegård et al., Phys. Rev. Lett. 86, 5866 (2001).

[5] J. Simpson, Z. Phys. A 358, 139 (1997).

[6] D. C. Radford, Nucl. Instrum. Methods Phys. Res., Sect. A 361, 297 (1995).

[7] I. Hamamoto, Phys. Rev. C 65, 044305 (2002). 